\title{
Colored Ring-Shaped Thread Tag Clippings to Identify the Accurate Location of Multiple Polyps Resected via Endoscopic Mucosal Resection
}

\author{
Hirohito Mori, Hideki Kobara, and Tsutomu Masaki \\ Department of Gastroenterology and Neurology, Kagawa University, Kagawa, Japan
}

Collection of multiple resected proximal colon polyps $>10$ $\mathrm{mm}$ in size through a scope channel via aspiration following an endoscopic mucosal resection (EMR) is difficult; thus, polyps are currently all grasped at once using net forceps. However, the simultaneous collection by net forceps makes it difficult to identify the accurate locations of all resected polyps. ${ }^{1}$ Therefore, we aimed to investigate a novel technique to identify the accurate location of resected polyps using colored ring-threads.

A 58-year-old man was diagnosed with three ascending colon polyps approximately $10-\mathrm{mm}$ in diameter. These polyps were located in the proximal colon and had the potential for

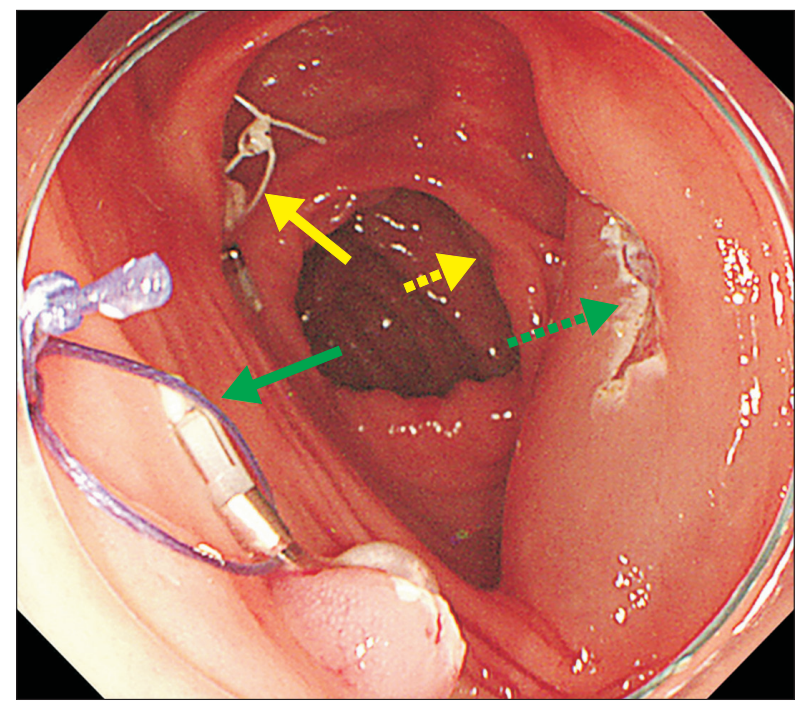

Fig. 1. Colored ring-thread tag clipping technique. A white ringthread 8-mm in diameter through a scope channel was clipped and fixed close to the first polyp (yellow arrows). Endoscopic mucosal resection was performed on the second polyp by clipping a purple ring-thread (green arrow); thus, the accurate locations of the resected polyps were obvious not only for the second resected polyp (green dotted arrow) but also the first polyp (yellow dotted arrow). cancerous components. The polyps were found to have an irregular microvascular pattern via narrow-band imaging magnified endoscopy. Thus, confirmation of the accurate location of each polyp was necessary because of the possibilities of subsequent additional EMR and surgery. Therefore, slight tattooing was performed in close proximity to each polyp. We prepared three types of colored and six sized ring-threads, such as white, purple, and black. After the local injection was performed and sufficient bulging was obtained, a white ring-thread 5-mm in diameter was clipped through the scope channel and fixed close to the first polyp. EMR was performed and included the clip

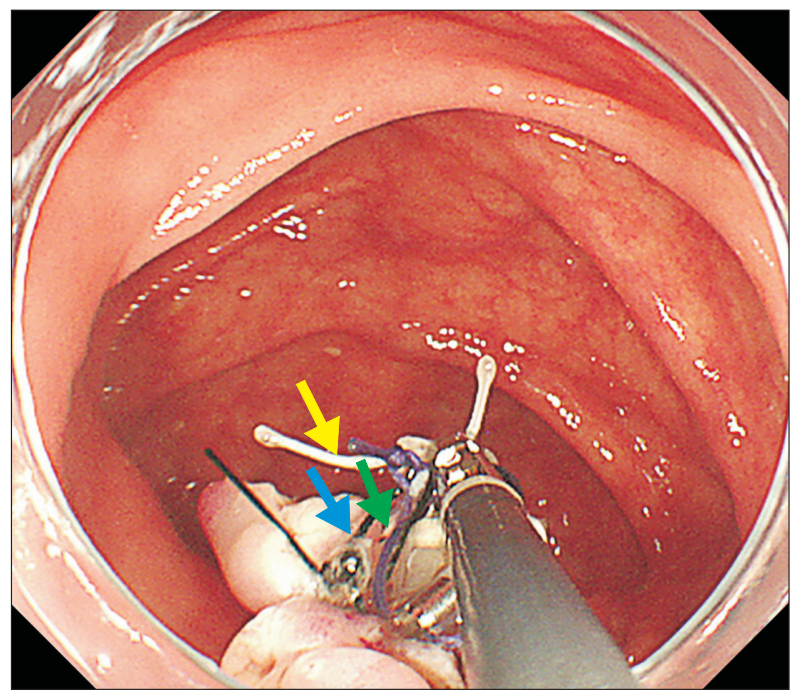

Fig. 2. Collection by hooking three colored ring-threads with grasping forceps. The 1st, 2nd, and 3rd polyps were marked with a black ring-thread. The resected polyps were lying on the ascending colon and were easily collected by hooking three colored ring-threads with grasping forceps (yellow, green, and blue arrows).

\section{Correspondence to: Hirohito Mori}

Department of Gastroenterology and Neurology, Kagawa University, 1750-1 Ikenobe, Miki-cho, Kita, Kagawa 761-0793, Japan

Tel: +81-87-891-2156, Fax: +81-87-891-2158, E-mail: hiro4884@med.kagawa-u.ac.jp

Received on April 14, 2016. Revised on May 12, 2016. Accepted on May 13, 2016. Published online October 13, 2016

pISSN 1976-2283 eISSN 2005-1212 https://doi.org/10.5009/gnl16197

@ This is an Open Access article distributed under the terms of the Creative Commons Attribution Non-Commercial License (http://creativecommons.org/licenses/by-nc/4.0) which permits unrestricted non-commercial use, distribution, and reproduction in any medium, provided the original work is properly cited. 
with the white ring-thread. Similarly, EMR was performed on the second and third polyps with a purple ring-thread (Fig. 1) and black ring-thread, respectively. Following the completion of all EMRs, grasping forceps were inserted through the scope channel to hook and grasp the colored ring-threads to retrieve all resected polyps (Fig. 2).

EMR has been widely implemented since it was established as a standard resection method for colorectal polyps. However, local recurrence is also reported in 13\% of cases. ${ }^{1}$ When multiple EMRs were performed with all polyps grasped and retrieved by net forceps at once, accurate identification of the resection sites was difficult. If the surgical margin-positive cancerous component remained among the resected specimens, specifying the accurate location of an additional resection would be difficult., This report suggested that a colored ring-thread tag clip technique with pre-EMR tattooing comprises a better approach to identify the accurate locations of resected polyps less than 20$\mathrm{mm}$ in size by EMR, as well as to subsequently collect all specimens completely. Moreover, this method is simple, and only 1 minute is required to put the ring-shaped thread close to polyp and clip it.

\section{CONFLICTS OF INTEREST}

No potential conflict of interest relevant to this article was reported.

\section{REFERENCES}

1. Fujita M, Tsuruta O, Ikeda H, Toyonaga A, Tanikawa K. Local recurrence of colorectal tumors after endoscopic mucosal resection. Int J Oncol 1997;11:533-538.

2. Carvalho R, Areia M, Brito D, Saraiva S, Alves S, Cadime AT. Endoscopic mucosal resection of large colorectal polyps: prospective evaluation of recurrence and complications. Acta Gastroenterol Belg 2013;76:225-230.

3. Park JJ, Cheon JH, Kwon JE, et al. Clinical outcomes and factors related to resectability and curability of EMR for early colorectal cancer. Gastrointest Endosc 2011;74:1337-1346. 